\title{
EUS-guided pancreatic radiofrequency ablation: preclinical comparison of two currently available devices in a pig model
}

\section{(ㄷ)(우우}

\author{
Authors \\ Frédéric Prat ${ }^{1,2}$ \\ Institutions \\ 1 Department of Gastroenterology, Cochin Hospital, \\ Assistance Publique-Hôpitaux de Paris, Paris, France \\ 2 Paris-Descartes University, Paris, France \\ 3 Department of Pathology, Cochin Hospital, Paris, France
}

Maximilien Barret ${ }^{1,2}$, Sarah Leblanc ${ }^{1,2}$, Alexandre Rouquette ${ }^{3}$, Stanislas Chaussade ${ }^{1,2}$, Benoit Terris ${ }^{2,3}$,

submitted 20.2.2018

accepted after revision 13.4 .2018

\author{
Bibliography \\ DOI https://doi.org/10.1055/a-0668-5653 | \\ Endoscopy International Open 2019; 07: E138-E143 \\ (c) Georg Thieme Verlag KG Stuttgart · New York \\ ISSN 2364-3722
}

Corresponding author

Frédéric Prat, Department of Gastroenterology, Cochin Hospital, 27 rue du Faubourg St Jacques, 75014 Paris

Fax: +33-1-58411965

frederic.prat@aphp.fr

\begin{abstract}
Introduction Two devices are currently available to perform pancreatic radiofrequency ablation (P-RFA). Potential clinical indications might extend from the treatment of pancreatic cystic lesions to ablation of small pancreatic solid lesions or cytoreduction of advanced pancreatic adenocarcinomas, but more preclinical data from animal models are needed to optimize P-RFA operation.
\end{abstract}

Methods P-RFA was performed under laparotomy and under endoscopic ultrasonographic guidance on the liver and pancreatic parenchyma of four live swine using the Habib EUS RFA (EMcision Ltd, London, UK) probe and the EUS-RA needle (Taewoong Medical, Gyeonggi-do, South Korea). Animals were sacrificed 2 hours after the procedure. Influence of tuning ablation time and power on tissue ablation were studied by histopathological assessment of the maximal depth of tissue damage on representative slides for each P-RFA shot.

Results The Habib probe in the liver parenchyma resulted in tissue necrosis increasing within the range of $1.9 \pm$ $0.5 \mathrm{~mm}$ (Power $=8 \mathrm{~W}$, Time $=120 \mathrm{~s}$ ) to $2.5 \pm 1 \mathrm{~mm}$ (Power $=$ $10 \mathrm{~W}$, Time $=120 \mathrm{~s}$ ). In the pancreatic parenchyma, tissue damage ranged from $3.1 \pm 0.4 \mathrm{~mm}$ (Power $=8 \mathrm{~W}$, Time $=$ $120 \mathrm{~s}$ ) to $2.3 \pm 0.1 \mathrm{~mm}(12 \mathrm{~W}, 120 \mathrm{~s})$ in depth. EUS RFA ablation of the liver parenchyma resulted in tissue damage ranging from $1.6 \pm 0.2 \mathrm{~mm}$ (Power $=30 \mathrm{~W}$, Time $=11 \mathrm{~s}$ ) to $1.5 \pm 0.1 \mathrm{~mm}$ (Power $=70 \mathrm{~W}$, Time $=9 \mathrm{~s}$ ); in the pancreas, ablation depth ranged from $3.6 \pm 0.5 \mathrm{~mm}$ (Power $=30 \mathrm{~W}$, Time $=15 \mathrm{~s})$ to $3.8 \pm 0.4 \mathrm{~mm}$ (Power $=70 \mathrm{~W}$, Time $=11 \mathrm{~s})$.

Conclusion Both devices allow for effective ablation of pancreatic tissue within 1.5 to $3.8 \mathrm{~mm}$ around the RFA electrode, with a modest influence of tuning power settings. Specific settings are recommended for each of the devices studied. Ablation of larger lesions may require more repeat P-RFA shots in different locations rather than a simple modulation of ablation parameters.

\section{Introduction}

Surgical pancreatic resection is currently considered the treatment of choice for most benign and malignant pancreatic tumors. However, pancreatic resection in expert centers bears a mortality rate of $0.6 \%$ to $1.4 \%$ and a severe morbidity rate of $16.6 \%$ to $19.8 \%$, with pancreatic fistula at the forefront [1]. Many pancreatic neoplasms with a low or moderate risk of malignancy, such as small pancreatic neuroendocrine tumors $(<2 \mathrm{~cm})$ or intraductal papillary mucinous neoplasms without high-risk stigmata are currently managed by surveillance instead of resection [2,3]. However, surveillance requires repeat magnetic resonance imaging procedures and follow-up endoscopic ultrasound (EUS), thereby incurring costs, psychological burden for patients and uncertain efficacy, furthermore depending on the patient's compliance. 
Pancreatic radiofrequency ablation (P-RFA) has been studied since 1999 and can be performed endoscopically using endoscopic ultrasound (EUS) guidance [4]. While clinical feasibility studies have already been conducted [5], the scope of the technique, which could extend from neuroendocrine tumors and intraductal papillary mucinous neoplasms (IPMN) to large lesions, including unresectable pancreatic adenocarcinoma, is as yet unclear [6]. Furthermore, different devices are currently available for EUS-guided pancreatic RFA, two of which, described below, have recently been made commercially available for clinical use. Because those devices use different technologies and instructions for use (IFU) are different, but are not supported by publically available preclinical data, we deemed it important to assess both devices with the same methodology in live animal models to verify the manufacturer's recommended settings and compare tissue ablation obtained.

\section{Methods}

\section{Animals}

Four landrace pigs weighing 30 to $35 \mathrm{~kg}$ stemming from the same farm were used for the study. Pigs were accommodated at our facility for 48 hours before procedure. Procedures were performed under general anesthesia. All animals were prepared for anesthesia with a 12-hour diet and administered an intramuscular injection of $10 \mathrm{mg} / \mathrm{kg}$ ketamine and $2 \mathrm{mg} / \mathrm{kg}$ azaperone 30 minutes before induction. After induction with $8 \mathrm{mg} / \mathrm{kg}$ intravenous (IV) $1 \%$ propofol and endotracheal intubation, anesthesia was maintained through inhalation of $2 \%$ isoflurane. All animals received an IV infusion of $10 \mathrm{mg} / \mathrm{kg} / \mathrm{h}$ crystalloid solution.

\section{Devices}

The Habib EUS RFA (EMcision Ltd, London, UK) device is a $1 \mathrm{Fr}$ wire monopolar electrode that can be inserted inside a standard 22G EUS fine-needle aspiration (FNA) needle, connected to a regular electrosurgical generator (ERBE Medizin VIO 200 or 300 series), and has no cooling system.

The EUSRA (Taewoong Medical, Gyeonggi-do, South Korea) is an 18G RFA needle, connected to a specific RF bipolar/monopolar VIVA RFTM generator associated with a pump cooling the needle internally with chilled saline solution.

\section{Experimental protocol}

The Habib EUS RFA and EUSRA probes were tested on the liver and pancreas of four animals with exactly the same methodology. Two animals were used to assess the effects of the Habib EUS RFA probe, and the other two pigs to assess the EUSRA device. A linear EUS probe (GFUCT140, Olympus, Tokyo, Japan) and a dedicated EUS platform (EUM2, Olympus, Tokyo, Japan) were used. The body of the pancreas was identified and punctured through the stomach. For the first pig, the Habib EUS RFA was inserted through a $22 \mathrm{G}$ EUS FNA needle (EchoTip Ultra, Cook, Limerick, Ireland). RF energy was delivered as recommended by the manufacturer for 120 seconds (Power $=10 \mathrm{~W}$, Effect $=4$ ) using a VIO $300 \mathrm{D}$ electrosurgical unit (ERBE Tubin- gen, Germany) to generate RF in the monopolar soft coagulation mode.

Then, a median laparotomy was performed. Three hepatic lesions were created with the Habib EUS RFA probe inserted into the left liver lobe parenchyma, using different ablation settings. For the second pig, after a median laparotomy, the hepatic ablations were repeated with the same three ablation settings as in the first one, and three lesions were created in the body and tail of the pancreas under direct visualization, with the same parameters and the same method as in the liver, each being identified by a stitch.

Procedures were the same for the next two pigs, except for RF settings with the EUSRA device: energy was delivered as recommended by the manufacturer at a power of $50 \mathrm{~W}$ for 15 seconds, using a VIVA RF generator and the procedure was

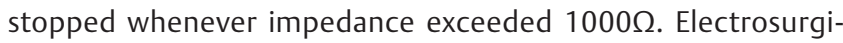
cal settings above and below IFUs were defined for both devices in accordance with the generator and probe manufacturers (Erbe Medical and EmCision, respectively, for the Habib device, Starmed for the EUS-RA device), with regard to the results of previous in vitro experiments to avoid tissue damage to adjacent organs and/or probe destruction.

Two hours after the procedure, with the animals still under general anesthesia, pigs were euthanized with $100 \mathrm{mg} / \mathrm{kg}$ IV pentobarbital. The liver, common bile duct, and duodenopancreatic region were collected en-bloc, fixed for 24 hours in 10 $\%$ buffered formalin. After macroscopic examination, ablated segments were embedded in paraffin, processed into $3-\mu \mathrm{m}$ thick sections, and stained with hematoxylin-eosin and saffron. Histological assessment was performed by a pathologist experienced in digestive pathology (AR), blinded to procedural data, using an ocular micrometer to measure the maximal extent of tissue necrosis in depth. Volumes were estimated postulating an ellipsoid form of the lesions, by the formula: $v=4 / 3 \times$ mabc (where $a$ is the length, $b$ the height and $c$ the width). The length was estimated by the length of the P-RFA probe $(10 \mathrm{~mm}$ for both EUS RFA and EUSRA), while the width and height were assumed to be equal and measured by the extent of tissue damage around the probe. The tissue was considered non-viable when it displayed a disorganized arrangement of cell layers, absence of normally shaped native cells, or condensed cell nucleus and cytoplasm suggesting cell death. Measurements were repeated on three representative slides for each condition of ablation. Statistical analysis was performed using Graphpad Software (GraphPad Software Inc., San Diego, California, United States). Results are expressed as mean \pm SD.

The experimental protocol was approved by the scientific committee of the Surgical School of Paris (Ecole de Chirurgie de l'Assistance Publique-Hôpitaux de Paris, France) and the experiments were performed according to the standard animal research guidelines established by the French Ministry of Agriculture. 

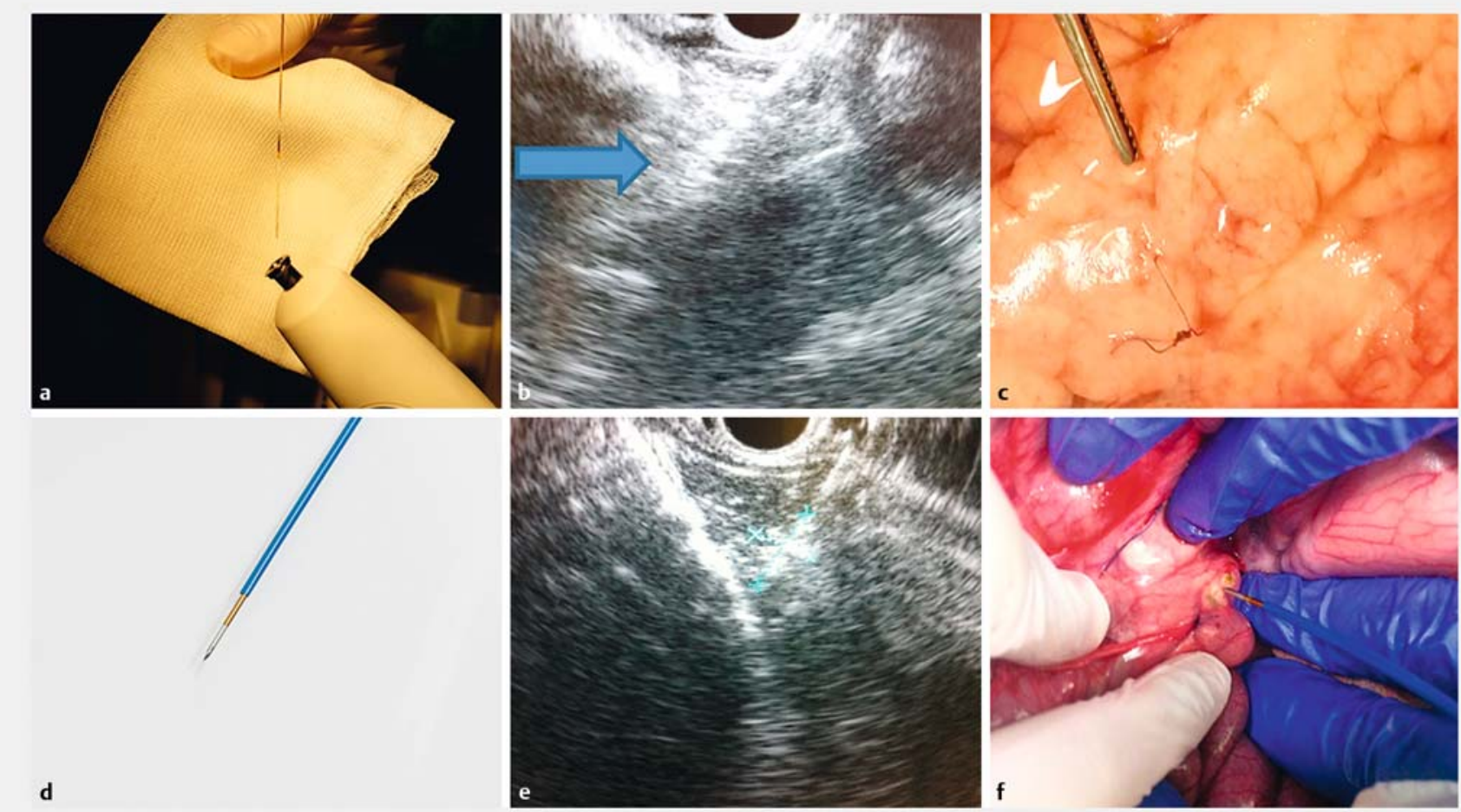

d

- Fig. 1 Pancreatic radiofrequency ablation using each of the two available probes. a Habib EUS RFA probe introduced into the EUS FNA. b EUS RFA using the Habib EUS RFA probe in healthy pancreatic body, with an echogenic cloud around the electrode. c Operative view of per-laparotomy radiofrequency ablation in the pancreas with the Habib EUS RFA probe. $\mathbf{d}$ EUSRA probe. e EUS-guided radiofrequency ablation using the EUSRA probe in healthy pancreatic body, with a echogenic cloud around the electrode. $f$ Operative view of per-laparotomy radiofrequency ablation in the pancreas using the EUSRA probe.

\section{Results}

P-RFA procedures under EUS guidance were technically successful with both the Habib EUS RFA and EUSRA devices, with adequate endosonographic visualization of the RFA probes and visualization of an echogenic cloud around the electrodes. However, the hyperechoic area was more clearly visible after application of the EUSRA probe. Representative views of the P-

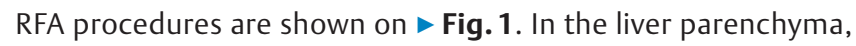
with the Habib EUS RFA probe, depth of tissue damage ranged from $1.9 \pm 0.5 \mathrm{~mm}$ for an ablation time of 120 seconds and a power setting of $8 \mathrm{~W}$, to $2.5 \pm 1 \mathrm{~mm}$ for an ablation time of 120 seconds and a power setting of $12 \mathrm{~W}$. Meanwhile, depth of tissue damage using the EUSRA probe ranged from $2.3 \pm 0.1 \mathrm{~mm}$ for an 11 -second, $30 \mathrm{~W}$ ablation to $1.5 \pm 0.1 \mathrm{~mm}$ for a 9 -second, $70 \mathrm{~W}$ ablation ( $>$ Table $\mathbf{1}$ ).

In the pancreas, depth of tissue damage ranged from $3.1 \pm$ $0.4 \mathrm{~mm}$ to $2.3 \pm 0.1 \mathrm{~mm}$ using the Habib EUS RFA probe with the same parameters as in the liver. With the EUSRA probe, depth of tissue damage ranged from $3.6 \pm 0.5 \mathrm{~mm}$ for a 15 -second and $30 \mathrm{~W}$ ablation to $3.8 \pm 0.4 \mathrm{~mm}$ for a 15 -second and $70 \mathrm{~W}$ ablation ( $\triangleright$ Table 2 ). The effect of increasing the power and time of ablation in the pancreatic parenchyma with each RFA probe is presented in > Fig. $\mathbf{2 a}$. The potential volume of the target lesions is reported in $>$ Table $\mathbf{3}$ and $>$ Fig. $\mathbf{2 b}$, and ranged from $131 \pm 0.1 \mathrm{~mm}^{3}$ to $189 \pm 0.8 \mathrm{~mm}^{3}$ for the Habib EUS RFA probe, and $256 \pm 0.2 \mathrm{~mm}^{3}$ to $335 \pm 0.8 \mathrm{~mm}^{3}$ for the EUS RA probe.

\section{Discussion}

Development of EUS RFA opens new perspectives in management of pancreatic neoplasia. Preliminary feasibility studies of cytoreduction or debulking in locally advanced pancreatic adenocarcinoma have shown a significant effect in terms of tumor volume reduction, but did not improve survival $[7,8]$. Some recent data obtained in small series of patients suggest that morbidity associated with EUS RFA is acceptable, with a low rate of pancreatitis and pancreatic fistulae $[5,6,9]$. For benign or focal pancreatic lesions, such as pancreatic cystic lesions (intraductal papillary mucinous neoplasms or mucinous cystadenomas) or small, low-grade neuroendocrine tumors, RFA might become an alternative to surgical resection and possibly reduce the need for lifelong surveillance of patients [8-11]. Among existing devices for pancreatic RFA, one (Cryothermprobe, Erbe Elektromedizin $\mathrm{GmbH}$, Tübingen, Germany) has been studied experimentally and in small series of patients [7], but it is currently not commercially available. Because experimental data on clinically available devices (i.e. the Habib EUS RFA and the EUS-RA) are lacking, we deemed it important to compare those devices to assess their results in terms of depth and volumes of tissue ablation. By using several power and time settings in live 
- Table 1 Outcomes of radiofrequency ablation in the liver parenchyma using each of two EUS RFA probes

\begin{tabular}{|c|c|c|c|c|c|}
\hline \multicolumn{3}{|c|}{$\begin{array}{l}\text { Habib EUS RFA probe } \\
(\text { effect }=4)\end{array}$} & \multicolumn{3}{|c|}{ EUSRA probe } \\
\hline Power (W) & Time (s) & $\begin{array}{l}\text { Maximal extent of tissue necrosis, } \\
\text { mean } \pm S D(\mathrm{~mm})\end{array}$ & Power (W) & Time (s) & $\begin{array}{l}\text { Maximal extent of tissue necrosis, } \\
\text { mean } \pm S D(\mathrm{~mm})\end{array}$ \\
\hline 8 & 120 & $1.9 \pm 0.45$ & 30 & 11 & $1.57 \pm 0.25$ \\
\hline 10 & 120 & $2.46 \pm 1.04$ & 50 & 9 & $1.22 \pm 0.03$ \\
\hline 12 & 120 & $2.02 \pm 0.44$ & 70 & 9 & $1.55 \pm 0.12$ \\
\hline
\end{tabular}

- Table 2 Outcomes of RFA in the pancreatic parenchyma using each of two EUS RFA probes

\begin{tabular}{|c|c|c|c|c|c|}
\hline \multicolumn{3}{|c|}{$\begin{array}{l}\text { Habib EUS RFA probe } \\
(\text { effect }=4)\end{array}$} & \multicolumn{3}{|c|}{ EUSRA probe } \\
\hline Power (W) & Time (s) & $\begin{array}{l}\text { Maximal extent of tissue necrosis, } \\
\text { mean } \pm \text { SD }(\mathrm{mm})\end{array}$ & Power (W) & Time (s) & $\begin{array}{l}\text { Maximal extent of tissue necrosis, } \\
\text { mean } \pm S D(\mathrm{~mm})\end{array}$ \\
\hline 8 & 120 & $3.11 \pm 0.37$ & 30 & 15 & $3.62 \pm 0.48$ \\
\hline 10 & 120 & $3.11 \pm 0.19$ & 50 & 9 & $3.80 \pm 0.25$ \\
\hline \multirow[t]{2}{*}{12} & \multirow[t]{2}{*}{120} & \multirow[t]{2}{*}{$2.31 \pm 0.08$} & 50 & 15 & $3.78 \pm 0.1$ \\
\hline & & & 70 & 11 & $3.81 \pm 0.45$ \\
\hline
\end{tabular}

- Table 3 Potential size and volume of tissue ablation with a single pancreatic RFA shot depending on power settings for each device.

\begin{tabular}{|c|c|c|c|c|c|c|c|}
\hline \multicolumn{4}{|c|}{$\begin{array}{l}\text { Habib EUS RFA probe } \\
(\text { effect }=4)\end{array}$} & \multicolumn{4}{|c|}{ EUSRA probe } \\
\hline Power (W) & Time (s) & $\begin{array}{l}\text { Mean lesion } \\
\text { diameter }(\mathrm{mm})\end{array}$ & $\begin{array}{l}\text { Mean lesion } \\
\text { volume }\left(\mathrm{mm}^{3}\right)\end{array}$ & Power (W) & Time (s) & $\begin{array}{l}\text { Mean lesion } \\
\text { diameter }(\mathrm{mm})\end{array}$ & $\begin{array}{l}\text { Mean lesion } \\
\text { volume }\left(\mathrm{mm}^{3}\right)\end{array}$ \\
\hline 8 & 120 & 6 & 189 & 30 & 15 & 7 & 256 \\
\hline 10 & 120 & 6 & 189 & 50 & 9 & 7.6 & 302 \\
\hline \multirow[t]{2}{*}{12} & \multirow[t]{2}{*}{120} & \multirow[t]{2}{*}{5} & \multirow[t]{2}{*}{131} & 50 & 15 & 7.6 & 302 \\
\hline & & & & 70 & 11 & 8 & 335 \\
\hline
\end{tabular}

pigs, we found with the Habib EUS RFA probe that the depth of tissue damage in the liver was proportional to the power used, and reached a radius of about $2.5 \mathrm{~mm}$ around the needle for a power of $10 \mathrm{~W}$, allowing for a treatment of 5- to 6-mm lesions, such as cystic lesions after the aspiration of cystic fluid. Similarly, in the pancreas, depth of tissue damage reached a ceiling, and was weakly influenced by the power variation beyond $10 \mathrm{~W}$, which does not support use of high-power settings with a 120 -second ablation time, as already suggested by Yoon et al [12]. With the EUSRA ${ }^{\mathrm{TM}}$ probe, depth of ablation varied along with ablation time, and was poorly influenced by high-power settings in the liver. Conversely in the pancreas, increasing time of ablation did not change depth of ablation at a power of $50 \mathrm{~W}$, but increasing power from 30 to $70 \mathrm{~W}$ linearly in- creased depth of ablation. Finally, a 15-second ablation time with a $30 \mathrm{~W}$ power setting would already allow for ablation of a 7-mm lesion.

As already suggested by others with the Habib EUS RFA probe [12] and by Kim et al. for the EUSRA electrode [13], elevated power settings do not increase depth of tissue damage around the radiofrequency electrode, because higher electrode temperature induces tissue charring, raising tissue impedance and reducing coagulative necrosis. However, this effect can be observed in a distinctive manner with each of the devices: because the Habib probe is much slimmer and has no internal cooling, it needs to be operated at a lower power and a longer exposure than the EUSRA probe, which can deliver more power in a shorter burst. With both probes, the small vol- 


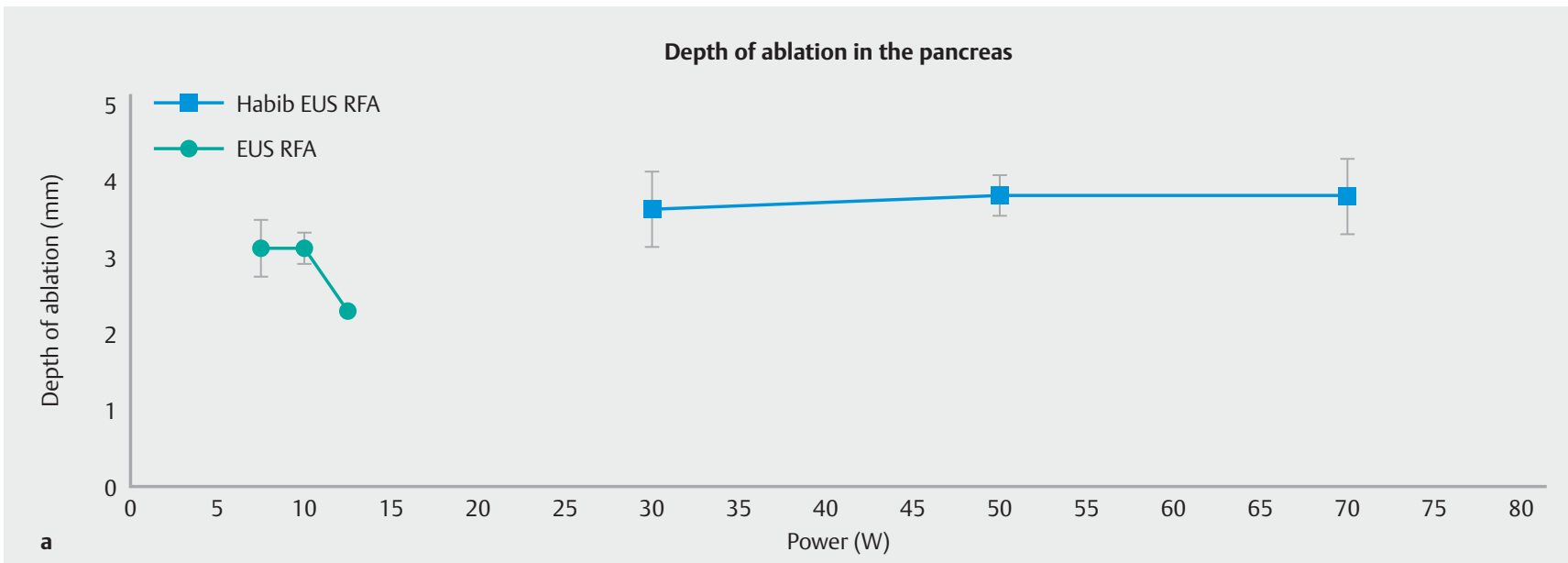

a

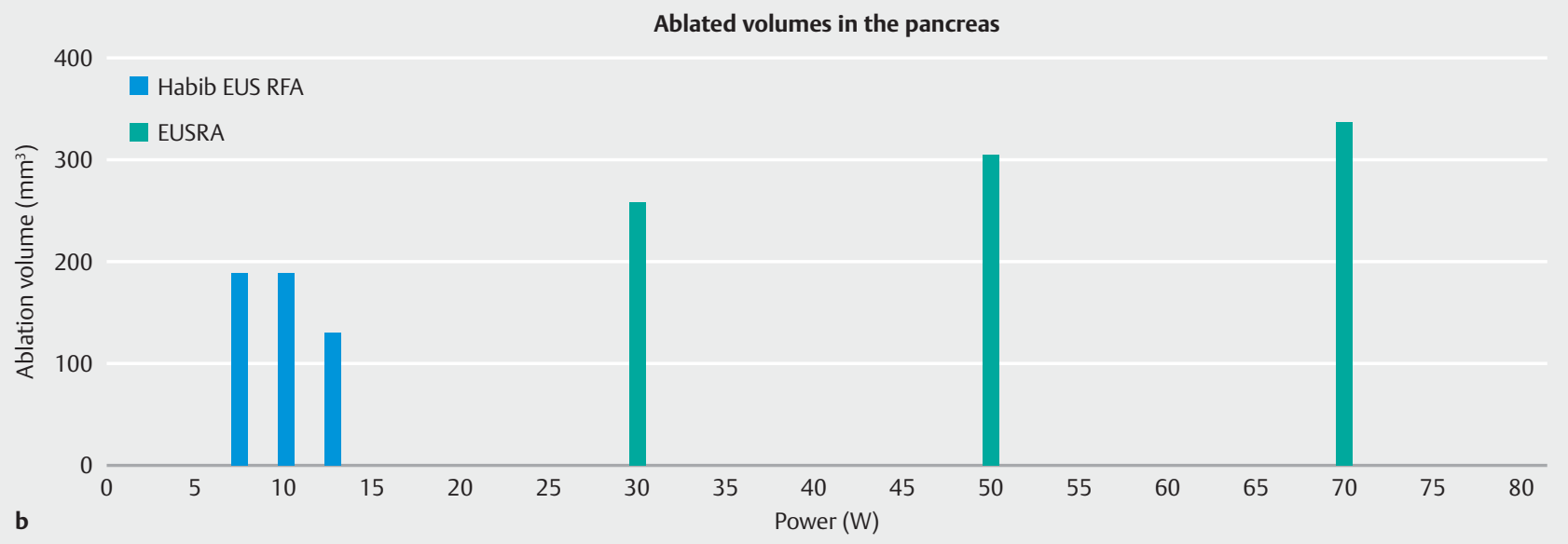

- Fig. 2 a Impact of power variation on the depth of ablated tissue in the pancreatic parenchyma with the Habib EUS RFA probe (time of ablation $=120$ seconds) and with the EUSRA probe (time of ablation of 15 seconds for 30 and $50 \mathrm{~W}$, and 9 seconds for $70 \mathrm{~W}$ ablation). $\mathbf{b}$ Impact of power variation on the volume of ablated tissue in the pancreatic parenchyma with the Habib EUS RFA probe (time of ablation=120 seconds) and with the EUSRA probe (time of ablation of 15 seconds for 30 and $50 \mathrm{~W}$, and 9 seconds for $70 \mathrm{~W}$ ablation).

ume of ablated tissue should be sufficient to treat a cystic lesion after aspiration of cystic fluid, but would require several treatment sessions for larger solid lesions [9]. Conversely, with the EUSRA device, the cooling system probably accounts for the absence of plateau in ablation depth when raising the power settings.

The example of the first generation of pancreatic radiofrequency electrodes used in the preoperative setting and their high rate of complications [14] should lead us to careful preclinical evaluation before implementation in clinical practice. Although other preclinical studies have already been conducted in the field, they either lacked measurement of tissue damage, used unavailable devices $[4,7]$, single generator settings with late assessment of tissue damage $[13,15]$, or reported results of only one RFA electrode [12].

This study is the first to compare several ablation settings with both commercially available P-RFA devices in a pig model. Furthermore, we assessed both EUS RFA and per laparotomy and "open" pancreatic RFA, which had only been done with the Habib EUS RFA probe [12]. The choice of performing only one EUS-guided ablation per animal and device and several un- der laparotomy was guided by the need first to demonstrate equivalent visibility and feasibility of RFA application in real conditions, which did not require repeating experiments; second, to correlate the puncture site with lesion location at sacrifice after repeat experiments, which could be done only under direct visualization.

The limitations of the current study include the small number of animals, with subsequently a limited set of ablation parameters, limited ability to explore the ablative effects in the full range of power and time settings, absence of well-defined pancreatic targets, and a short-term observation period. We acknowledge that a much higher number of experiments could make the findings stronger, but we considered that the probability that sacrificing many more animals would significantly change our results was low, considering the strict conditions of the experiments and the good reproducibility of results for most of the conditions tested. Exploring a wider range of parameters would have also required many more animals, and we found it questionable to depart greatly from the settings recommended by the companies as a starting point for our tests, because the risk of major damage or absence of lesions would 
likely be seriously and unacceptably increased. Regarding the short observation between P-RFA and sacrifice, although it is well known that full necrosis, scarring, and fibrosis may need several days and weeks to consolidate, it has also been shown that immediate macroscopic and histopathologic analysis provides an excellent proxy for the extent of definitive tissue damage [16].

Our results show effective ablation of pancreatic tissue about $2.5 \mathrm{~mm}$ around the RFA electrode with both devices. Both electrodes showed a high therapeutic index in the pancreas and liver, with little influence of increasing the power settings on the depth of tissue damage. These data support use of $10 \mathrm{~W}, 120$-second ablation settings for the Habib EUS RFA probe and $30 \mathrm{~W}, 15$-second settings for the EUSRA when performing EUS RFA for pancreatic lesions of 5 to $6 \mathrm{~mm}$. Ablation of larger lesions should probably require repeated procedures rather than modifications of the ablation parameters.

Finally, beyond the differences in technology and instructions for use, each device has some pros and cons that must be acknowledged, about which we can provide a couple of hints. One is the ability of the probe to sustain several applications in the same patient with the same efficacy in the case of a large tumor. While waiting for completion of RFA, the endosonographer needs to keep the electrode in the same position. But the fiber-like thin Habib probe, once exposed outside of the FNA needle, may not be strong enough to keep its original axis and shape and can bend because of the patient's respiration and aortic pulsations, making further ablations more difficult than with EUSRA. On the other hand, another important issue is the access of P-RFA to targets disseminated in the whole pancreatic gland, with some areas, such as the uncinate process, the pancreatic flexure of the very tip of the tail, being well-known for a more difficult access. It must be noted that the Habib probe, after insertion into a standard 22G needle, presents the same stiffness as the regular stylet, whereas the stiffer 18G EUSRA can be more difficult to handle in some challenging areas of the pancreas and be at a disadvantage in such cases. It is difficult from our study to draw any conclusion regarding those two issues, because ablations were done under either laparoscopic or EUS guidance in an easy-to-reach part of the pancreatic body.

\section{Conclusion}

Only clinical experience will provide adequate insights into the optimal choice and handling of P-RFA devices, but we hope these preclinical data can help design future clinical studies more robustly.

\section{Acknowledgements}

The authors wish to thank Life Partners Europe (Bagnolet, France) and Taewoong Medical, (Gyeonggi-do, Korea) for their assistance and the Surgical School of the Assistance PubliqueHôpitaux de Paris.
Competing interests

None

References

[1] McMillan MT, Christein JD, Callery MP et al. Comparing the burden of pancreatic fistulas after pancreatoduodenectomy and distal pancreatectomy. Surgery 2016; 159: 1013-1022

[2] Falconi M, Eriksson B, Kaltsas G et al. Vienna Consensus Conference participants. ENETS Consensus Guidelines Update for the Management of Patients with Functional Pancreatic Neuroendocrine Tumors and Non-Functional Pancreatic Neuroendocrine Tumors. Neuroendocrinology 2016; 103: $153-171$

[3] Tanaka M, Fernández-del CastilloC, Adsay V et al. International Association of Pancreatology. International consensus guidelines 2012 for the management of IPMN and MCN of the pancreas. Pancreatology 2012; 12: $183-197$

[4] Goldberg SN, Mallery S, Gazelle GS et al. EUS-guided radiofrequency ablation in the pancreas: results in a porcine model. Gastrointest Endosc 1999; 50: $392-401$

[5] Rustagi T, Chhoda A. Endoscopic radiofrequency ablation of the pancreas. Dig Dis Sci 2017; 62: 843-850

[6] Signoretti M, Valente R, Repici A et al. Endoscopy-guided ablation of pancreatic lesions: Technical possibilities and clinical outlook. World ] Gastrointest Endosc 2017; 9: 41 - 54

[7] Arcidiacono PG, Carrara S, Reni M et al. Feasibility and safety of EUSguided cryothermal ablation in patients with locally advanced pancreatic cancer. Gastrointest Endosc 2012; 76: $1142-1151$

[8] Song TJ, Seo DW, Lakhtakia S et al. Initial experience of EUS guided radiofrequency ablation of unresectable pancreatic cancer. Gastrointest Endosc 2015; 83: 440 - 443

[9] Pai M, Habib N, Senturk $\mathrm{H}$ et al. Endoscopic ultrasound guidedradiofrequency ablation, for pancreatic cystic neoplasms and neuroendocrine tumors. World J Gastrointest Surg 2015; 7: 52 - 59

[10] Waung J, Todd JF, Keane MG et al. Successful management of sporadic pancreatic insulinoma by endoscopic ultrasound-guided radiofrequency ablation. Endoscopy 2016; 48: E144-E145

[11] Rossi S, Viera FT, Ghittoni G et al. Radiofrequency ablation of pancreatic neuroendocrine tumors: a pilot study of feasibility, efficacy, and safety. Pancreas 2014; 43: 938 - 945

[12] Yoon WJ, Daglilar ES, Kamionek M et al. Evaluation of radiofrequency ablation using the 1-Fr wire electrode in the porcine pancreas, liver, gallbladder, spleen, kidney, stomach, and lymph nodes: A pilot study. Dig Endosc; 2015: doi:10.1111/den.12575 [Epub ahead of print]

[13] Kim HJ, Seo DW, Hassanuddin A et al. EUS-guided radiofrequency ablation of the porcine pancreas. Gastrointest Endosc 2012; 76: $1039-1043$

[14] Wu Y, Tang Z, Fang $\mathrm{H}$ et al. High operative risk of cool-tip radiofrequency ablation for unresectable pancreatic head cancer. J Surg Oncol 2006; 94: $392-395$

[15] Silviu UB, Daniel P, Claudiu M et al. Endoscopic ultrasound-guided radiofrequency ablation of the pancreas: An experimental study with pathological correlation. Endosc Ultrasound 2015; 4: 330 - 335

[16] McGahan JP, Brock JM, Tesluk $\mathrm{H}$ et al. Hepatic ablation with use of radio-frequency electrocautery in the animal model. J Vasc Interv Radiol 1992; 3: $291-297$ 